\section{Implicancias en la actividad física y la salud del Programa CicloRecreoVía en Chile}

\section{Physical activity and health implications of the CicloRecreoVia program in Chile}

Background: With approximately 66\% of the population overweight and nearly $90 \%$ with sedentary lifestyles, obesity has become a major public health problem in Chile. Thus, in the last fifteen years health policies have promoted active lifestyles. An interesting initiative in this context is the CicloRecreoVia program, which closes a set of public streets on Sunday mornings, allowing people to carry out PA in streets free of vehicles. Aim: To characterize PA and mobility patterns of participants of the CicloRecreoVia program in Santiago. Material and Methods: 401 participants of five circuits of the CicloRecreoVia surveyed on the type, frequency and durantion of the PA carried out, their daily mobility habits, motivations for attending the CicloRecreoVia, self-perceived health condition, among other aspects. Results: Participants spent 113 minutes in the CicloRecreoVia, 77\% declared walking or bicycling at least 10 minutes per day and $56.7 \%$ traveled actively (bicycling or walking) at least four times per week. Finally, 69.1\% perceived their health condition "better" or "much better" than one year ago. In addition, it was noted that CicloRecreoVias attracted different type of participants depending on their location in the city. Conclusions: In line with previous studies, most users achieve about $75 \%$ of the weekly PA recommended by attending the CicloRecreoVia, and the main motivations to attend the program were to make $P A$, to be outdoor and to socialize. As a result, the CicloRecreoVia has the potential to become an effective public nationwide health policy to combat obesity.

(Rev Med Chile 2018; 146: 451-459)

Key words: Ciclorecreovia Program; physical activity, Santiago.
'Escuela de Arquitectura, Facultad de Arquitectura, Arte y Diseño, Universidad Diego Portales. Santiago, Chile. ${ }^{2}$ Centro para el Desarrollo Urbano Sustentable, CEDEUS.

${ }^{3}$ Escuela de Arquitectura, Pontificia Universidad Católica de Chile. Santiago, Chile.

${ }^{\text {aAqquitecto. }}$

${ }^{\text {b} A r q u i t e c t o, ~ M a g i ́ s t e r ~ e n ~}$ Sociología.

'Arquitecto, Magíster en Arquitectura.

${ }^{\mathrm{d} M S C}$.

ePhD.

Fuente de apoyo: Los autores agradecen el apoyo del proyecto FONDECYT 1171232 y de CEDEUS (FONDAP 15110020) por haber financiado parcialmente este trabajo.

Recibido el 29 de agosto de 2017, aceptado el 11 de abril de 2018.

Correspondencia a: Rodrigo Mora Escuela de Arquitectura, Facultad de Arquitectura, Arte y Diseño, Universidad Diego Portales. República 180, Santiago centro, Santiago. Teléfono: 226762702 rodrigo.mora@udp.cl
L a última Encuesta Nacional de Salud ${ }^{1}$ (ENS) mostró que nueve de cada diez chilenos no realizaba actividad física (AF) en forma regular. Estas cifras, sumadas al creciente sobrepeso que afecta a casi dos de cada tres chilenos ${ }^{2}$, llevaron al Ministerio de Salud a iniciar diferentes iniciativas de salud preventiva a finales de los años noventa. En 1998 se lanzó el Plan Nacional de Promoción de la Salud ${ }^{3}$, que en 2004 se transformó en la "Estrategia Global contra la Obesidad" $(\mathrm{EGO})^{4}$, iniciativas que buscaron modificar los hábitos alimenticios y fomentar la realización de AF por parte de los habitantes. En 2007, la EGO es reemplazada por el programa Elige Vivir Sano ${ }^{5}$, que junto con mantener las recomendaciones en torno al aspecto nutricional del problema de la obesidad, puso el acento en la necesidad de integrar la AF a la rutina de las personas. Cuatro años más tarde, la Estrategia Nacional de Salud ${ }^{6}$, fija dentro de sus metas bajar la prevalencia de obesidad de $9,6 \%$ a $8,6 \%$, y aumentar la práctica de AF en jóvenes de $23,1 \%$ a $30 \%$. 
A pesar de lo anterior, estos esfuerzos no han tenido una mirada global sobre la ciudad ${ }^{7,8}$, y específicamente sobre los efectos que el medio urbano puede tener en las personas para fomentar la adopción de modos de vida activos en las rutinas de las personas ${ }^{9,10}$, como irse en bicicleta o caminando a los lugares de trabajo o estudio. En efecto, las Metas Sanitarias de la Estrategia Nacional de Salud ${ }^{6}$ hacen una mención muy menor al medio urbano como variable interviniente para el fomento de los modos de vida activos. Así, a diferencia de lo que ocurre en países desarrollados, donde el combate a la obesidad tiene como protagonistas a expertos del área de la salud, el transporte y la planificación urbana $^{11-14}$, en Chile estas políticas aún no integran completamente a la ciudad.

Un excepción a esta tendencia lo constituye el programa CicloRecreoVía, que consiste en el cierre temporal de calles a los autos para incentivar el uso de la bicicleta, la caminata, el trote, la patineta y otras actividades en el espacio público. Originario de Colombia, donde tiene el nombre de Ciclovía Recreativa, este programa forma parte de un conjunto de iniciativas colombianas que han sido incorporadas a la planificación urbana a nivel mundial ${ }^{15}$.

\section{El programa de la CicloRecreoVía}

La Ciclovía Recreativa comenzó su funcionamiento en Bogotá en 1974, como iniciativa promovida para fomentar el uso de la bicicleta ${ }^{16}$. Hasta inicios de los noventa este programa tuvo una continuidad fluctuante y operó en forma más bien discreta. Sin embargo, la llegada de los alcaldes Mockus (1995-1997, 2001-2003) y Peñalosa (1998-2000), significó la expansión a gran escala de esta iniciativa, quienes vieron en el programa una oportunidad para recuperar el espacio público para los habitantes, en un contexto político complejo y con la creciente amenaza del narcotráfico ${ }^{17}$. Actualmente en Colombia todos los domingos se abren 121 kilómetros de calles entre las 07:00 y las 14:00 h, permitiendo que entre 600.000 y 1.400 .000 personas, la mayor parte de estratos pobres, caminen, patinen y pedaleen ${ }^{18}$.

A principios de la década pasada comienza la expansión internacional de la Ciclovía Recreati$\mathrm{va}^{17}$. Ayudado por una buena cobertura mediática y la existencia de buenos "embajadores" de la política urbana colombiana ${ }^{17}$, este programa es rápidamente adoptado por numerosas ciudades latinoamericanas y norteamericanas. Actualmente 33 ciudades $^{18}$ en 27 países diferentes (la mayor parte en el continente americano), tienen este tipo de programas en marcha, involucrando a más de 1,5 millones de personas por semana ${ }^{16}$. Más aún, ciudades tradicionalmente dominadas por el auto, como la ciudad de Los Ángeles, en Estados Unidos de Norteamérica, han adoptado el cierre temporal de calles para dar paso al uso temporal por parte de los ciudadanos ${ }^{19,20}$.

Estudios internacionales han mostrado que más de $90 \%$ de los usuarios de este tipo de programas realizan más de 30 min de actividad física, y que entre $40 \%$ y $90 \%$ de los participantes cumple en el día de la CicloRecreoVía los 150 min de actividad física moderada a la semana recomendada por la $\mathrm{OMS}^{21}$. Asimismo, investigaciones realizadas en Colombia han mostrado que este tipo de programas suele estar acompañado de clases de educación física ${ }^{16}$ y que se ubican cercanos a ciclovías o parques, lo que promueve el uso de la bicicleta o la caminata para acceder a ellos $^{21}$. Conjuntamente a sus efectos en la salud, el carácter familiar y recreativo de este tipo de programas han incentivado el uso de la bicicleta y la caminata ${ }^{21}$. Finalmente, estudios realizados en Colombia muestran que este tipo de programas es beneficioso económicamente, toda vez que producen beneficios en salud equivalentes a entre 2 y 3 veces sus costos ${ }^{22}$.

En Chile, este programa se inició en 2007 en la comuna santiaguina de San Bernardo, operando actualmente en siete comunas capitalinas (Figura 1), además de las ciudades de Concepción y Antofagasta ${ }^{23}$. Actualmente se cierran cada semana a los autos 33 kilómetros de calles, lo que permite la participación semanal de cerca de 30.000 personas $^{23}$. A diferencia de Colombia donde forma parte de los programas de salud preventiva financiandos y administrados por el Ministerio de Salud, en Chile este programa ha tenido un apoyo financiero intermitente del gobierno y opera bajo el alero de la consultora privada Geomás, que vela por el cierre de calles y la administración de contratos publicitarios con auspiciadores.

Con el objeto de entender las implicancias en la salud de este programa, a continuación se detallan los resultados de una encuesta aplicada a usuarios de la CicloRecreoVía en la ciudad de Santiago. 


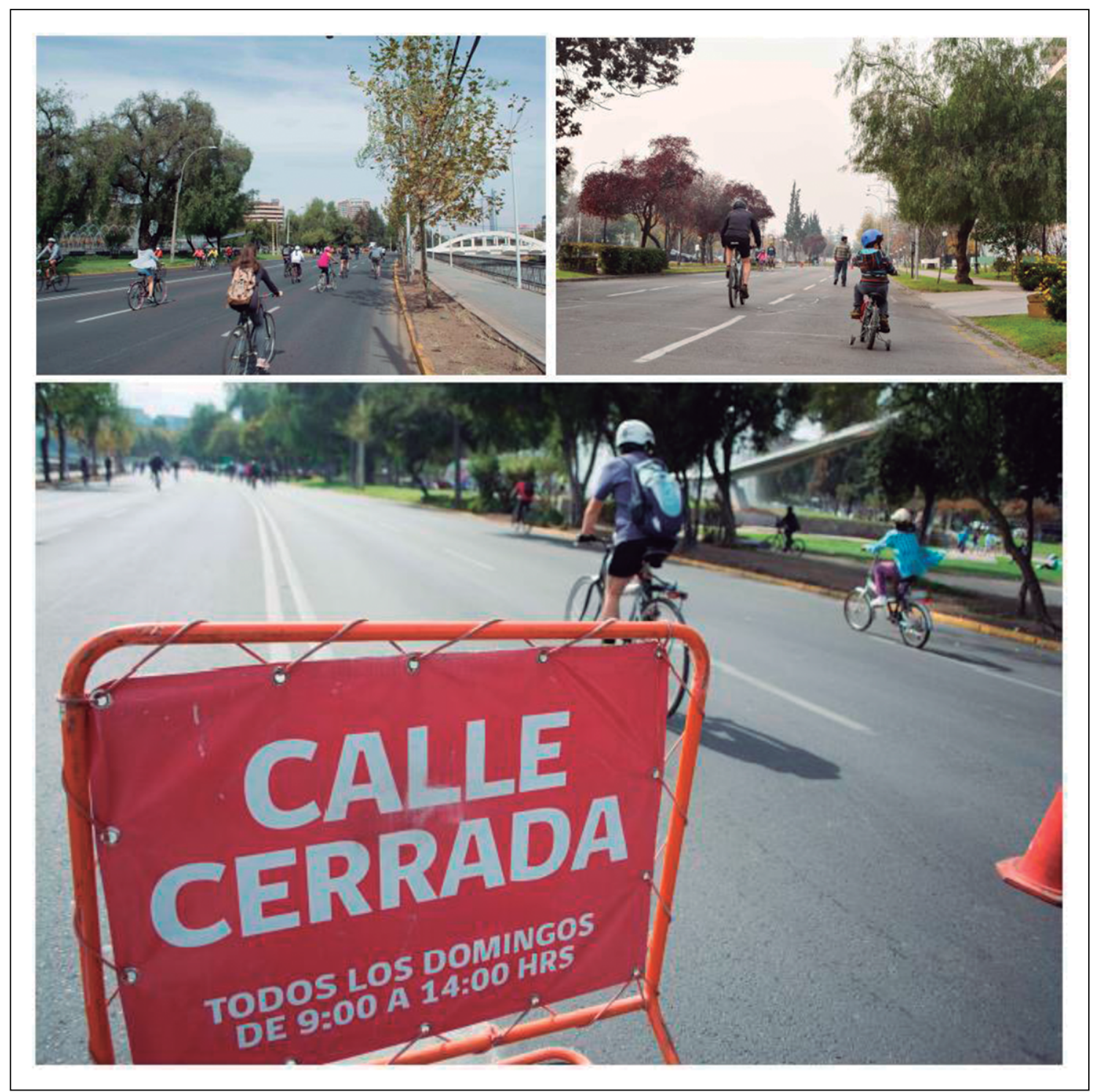

Figura 1. Actividad física en la CiclorecreoVía. Fuente: Manfredo Corado.

\section{Material y Método}

Para realizar la investigación se diseñó y aplicó una encuesta a 401 personas aleatoriamente elegidas $(65 \%$ hombres, $35 \%$ mujeres, edad promedio 37,2 años, DE = 14,2), de cinco CicloRecreoVías santiaguinas ubicadas en comunas de diferente nivel socioeconómico: Andrés Bello (nivel medio y medio alto, comunas de Santiago y Providencia), Pocuro-Lyon (nivel medio-alto, comuna de Pro- videncia), La Florida (nivel medio y medio-bajo, comuna de La Florida), Santiago centro (nivel medio y medio-bajo, comunas de Santiago y Recoleta) e Irarrázaval (nivel medio y medio-alto, comuna de Ñuñoa). La Figura 2 muestra la ubicación de las cinco CicloRecreoVías estudiadas en esta investigación.

La encuesta contenía diecisiete preguntas en total y se basó en las encuestas diseñadas por Aaron Hipp, investigador de la North Carolina 


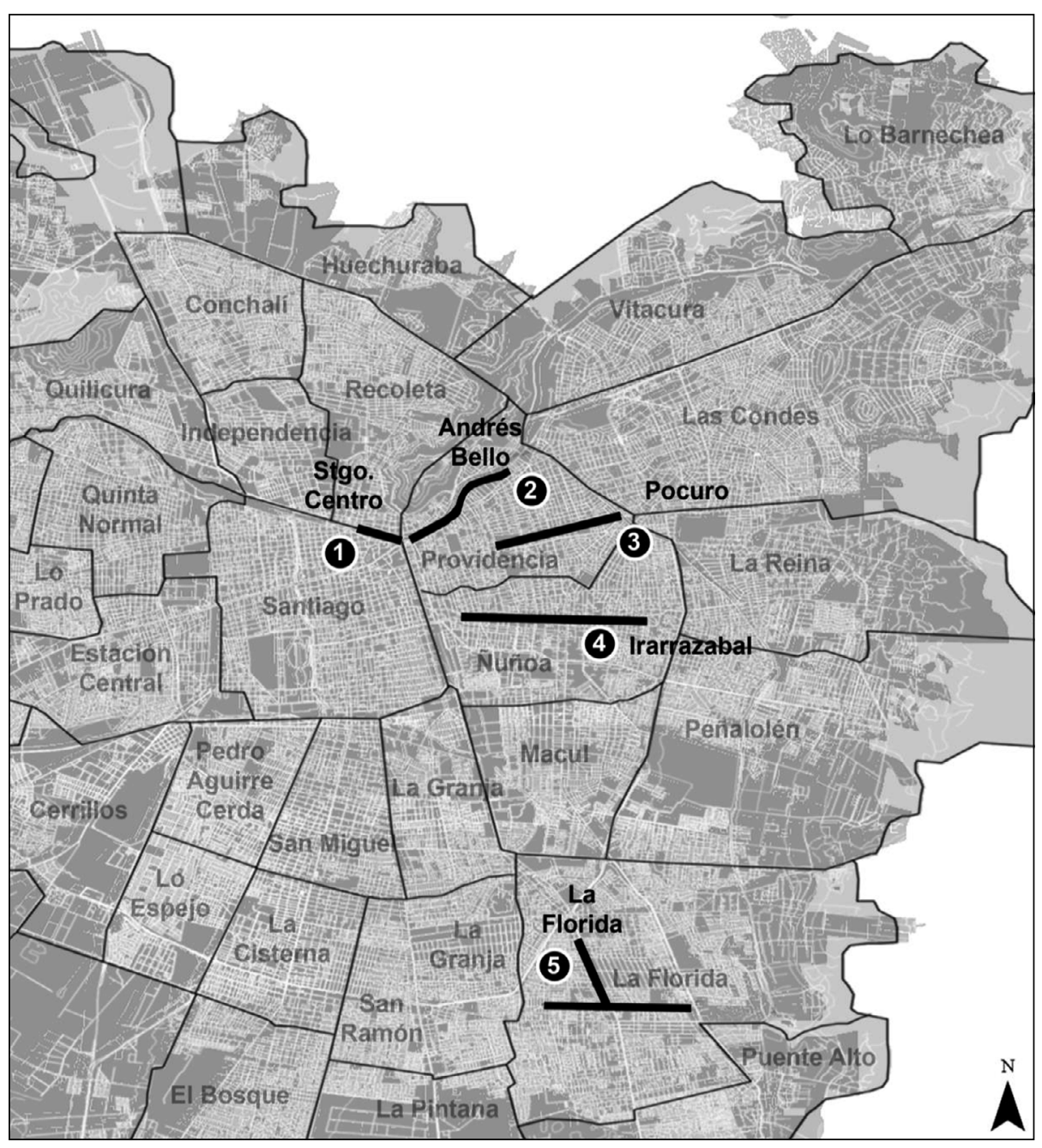

Figura 2. Las cinco CicloRecreoVías analizadas en esta investigación. Fuente: elaboración propia.
State University (disponible en la página de Active Living Research ${ }^{24}$ y el cuestionario Global Physical Activity Questionnaire, GPAQ ${ }^{25}$, instrumento internacionalmente validado para medir la actividad fisica urbana. Con el objeto de caracterizar socioeconómicamente el barrio donde vivían los encuestados, cada participante debió señalar la esquina más próxima de su casa. Estos datos fueron luego traspasados a una plataforma de Sistema de Información Geográfica (SIG) para su análisis.

Los encuestados fueron abordados en las estaciones de descanso dispuestas a lo largo de los circuitos. La encuesta demandaba entre cinco a diez minutos en ser respondida y fue realizada en los meses invernales de agosto y septiembre de 2016. Previo a la realización de la encuesta, los participantes firmaron un consentimiento informado visado por el Comité de Ética de la Pontificia Universidad Católica de Chile.

\section{Tamaño de la muestra}

Dado que no existía un número oficial de los participantes de las Ciclorecreovías, se asumió que la población era infinita y con varianza máxima. Asumiento una selección aleatoria simple de observaciones, se obtuvo un nivel de error de 4,3\%, con un intervalo de confianza de $95 \%$.

A continuación se presentan las preguntas relativas a salud y AF de la encuesta:

Familiaridad con el programa CicloRecreoVía. 


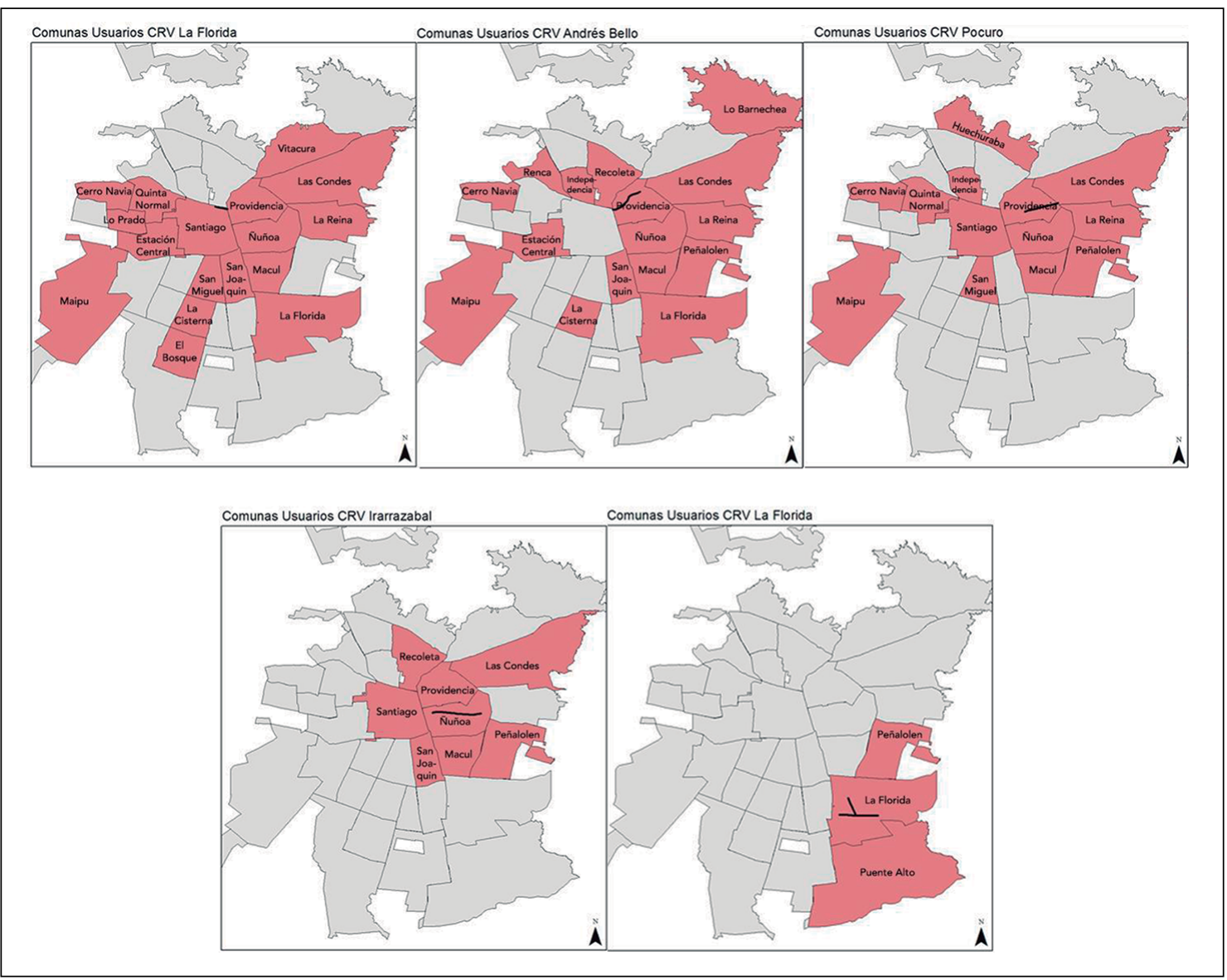

Figura 3. Procedencia comunal de los encuestados en cada CicloRecreoVía. Fuente: elaboración propia.

Se preguntó si era la primera vez que asistían al programa (sí/no), y cuántas veces habían asistido el último año (número de veces).

Actividad física realizada en la CicloRecreoVía. Se preguntó qué tipo de actividad era realizada habitualmente cuando asistían al programa (caminata/trote/bicicleta/andar en patines o skate/ otros), y cuánto tiempo consideraban pasar, o habían pasado, en el programa. No se discriminó por la intensidad de la actividad física.

Hábitos de movilidad diarios y auto-percepción de la salud. Se seleccionaron dos preguntas del GPAQ, y una pregunta sobre auto-percepción de la salud de la ENS 2009-10. Estas preguntas fueron: ¿camina o usa bicicleta usted en sus traslados cotidianos? (sí/no/no contesta), en una semana normal: ¿cuántos días a la semana camina o va en bicicleta en sus desplazamientos? (número de días), y finalmente ¿cómo diría que es su estado de salud comparado con el de hace un año? (mucho mejor/algo mejor/más o menos igual/algo peor/ mucho peor).

\section{Resultados}

La Tabla 1 expone las principales características de los encuestados. El 65\% de los encuestados fueron hombres, mientras que la edad promedio de los encuestados alcanzó 37,1 años. El 68,4\% de los que respondieron la encuesta dijeron que habitualmente usaban la CicloRecreoVía para andar en bicicleta, trotar $(18,1 \%)$, patinar o andar en skate (9,8\%), y finalmente la caminar $(3,7 \%)$.

Aunque la cantidad de encuestados no fue la misma en las CicloRecreoVías analizadas, los 
Tabla 1. Largo de las CicloRecreoVías analizadas y características de la muestra de encuestados

\begin{tabular}{|lccccc|c|}
\hline Circuito & $\begin{array}{c}\text { Longitud } \\
\text { circuito } \mathbf{( k m )}\end{array}$ & $\mathbf{n}$ & $\begin{array}{c}\text { Género } \\
(\% \mathbf{H} / \% \mathbf{M})\end{array}$ & $\begin{array}{c}\text { Edad } \\
\text { promedio }\end{array}$ & $\begin{array}{c}\text { Actividad principal } \\
\text { (\% Caminata / \% Trote / \% Bicicleta / \% Otros) }\end{array}$ \\
\hline 1. Santiago Centro & 2,6 & 79 & $84 / 16$ & 41 & $3 / 11 / 78 / 8$ \\
\hline 2. Andrés Bello & 4,1 & 127 & $63 / 37$ & 34 & $4 / 21 / 64 / 12$ \\
\hline 3. Pocuro & 2,3 & 81 & $56 / 44$ & 38 & $6 / 20 / 65 / 9$ \\
\hline 4. Irarrázaval & 3,9 & 63 & $52 / 48$ & 35 & $6 / 29 / 54 / 12$ \\
\hline 5. La Florida & 4,9 & 51 & $69 / 31$ & 39 & $0 / 6 / 88 / 6$ \\
\hline Total & 3,6 & 401 & $65 / 35$ & 37,1 & $3,7 / 18,1 / 68,4 / 9,8$ \\
\hline
\end{tabular}

Fuente: elaboración de los autores.

Tabla 2. Procedencia comunal de los encuestados y distancia promedio de éstos a la CicloRecreoVía elegida (diatancia Euclidiana)

\begin{tabular}{|llcc|}
\hline Circuito & & $\begin{array}{c}\text { Procedencia Comunal de los } \\
\text { encuestados } \\
\text { (cantidad de comunas) }\end{array}$ & $\begin{array}{c}\text { Distancia Promedio de los } \\
\text { encuestados al centro de la } \\
\text { CicloRecreoVía (en metros) }\end{array}$ \\
\hline Santiago Centro & $(n=78)$ & 17 & 5.042 \\
\hline Andrés Bello & $(n=80)$ & 13 & 3.677 \\
\hline Pocuro & $(n=125)$ & 16 & 4.208 \\
\hline Irarrázaval & $(n=49)$ & 3 & 2.661 \\
\hline La Florida & $(n=63)$ & 9 & 2.362 \\
\hline
\end{tabular}

Fuente: elaboración de los autores.

resultados sugieren que la ubicación de éstas en la ciudad tuvo efectos en el tipo de participante que acude a ellas. En efecto, las CicloRecreoVías ubicadas en lugares centrales como Andrés Bello y Santiago Centro, y en menor medida la de Pocuro, fueron visitadas por un mayor número de personas ajenas a la comuna respectiva; mientras que CicloRecreoVías localizadas en barrios residenciales no centrales como Irarrázaval o La Florida, fueron más bien de carácter local, esto es, convocaron mayoritariamente a vecinos de la misma comuna. Tal como puede verse en la Tabla 2, los encuestados de la CicloRecreoVía de Santiago Centro $(\mathrm{n}=78)$ fueron de 17 comunas diferentes, los participantes de la CicloRecreoVía de La Florida $(n=63)$, provinieron de 3 comunas. Esto es consistente con la distancia promedio que tuvieron los participantes de las CicloRecreoVías: mientras los participantes de Santiago Centro viajaban en promedio 5.042 metros para llegar al centro de esta CicloRecreoVía, los de La Florida solo necesitaban 2.661 metros para llegar a su centro. Estas diferencias no son menores y sugieren que las CicloRecreoVías tienen distinto carácter: mientras algunas son espacios de recreación y de encuentro metropolitano, otras operan más bien a nivel local como espacios para la realización de AF.

La Tabla 3 expone los resultados de las preguntas relacionadas con AF y salud en las CicloRecreoVías analizadas. Tal como puede observarse, el promedio de tiempo que las personas declararon practicar AF en la CicloRecreoVía fue de $113 \mathrm{~min}$, con un rango de entre 93 min (Irarrázaval) y 136 min (Santiago Centro). El promedio corresponde a cerca de $75 \%$ del tiempo recomendado por la Organización Mundial de la Salud ${ }^{7}$ como actividad física semanal. Nuevamente, se detectaron importantes diferencias en las CicloRecreoVías de carácter más local, como Irarrázaval y La Florida, donde las personas ocuparon 93 y 102 min respectivamente, versus las de carácter más metropolitano, como las de Santiago Centro y 
Tabla 3. Principales resultados de encuesta aplicada a participantes de la CicloRecreoVía

\begin{tabular}{|c|c|c|c|c|c|c|}
\hline Circuito & $\begin{array}{l}\text { Santiago } \\
\text { Centro }\end{array}$ & $\begin{array}{l}\text { Andrés } \\
\text { Bello }\end{array}$ & Pocuro & Irarrázaval & La Florida & Total \\
\hline $\begin{array}{l}\text { Encuestados que acuden por primera vez a } \\
\text { la CicloRecreoVía (\%) }\end{array}$ & 11,5 & 10,6 & 11,1 & 12,7 & 5,9 & 9,7 \\
\hline $\begin{array}{l}\text { Cantidad de tiempo que pretende pasar en } \\
\text { la CicloRecreoVía (en minutos) }\end{array}$ & 136 & 127 & 107 & 93 & 102 & 113 \\
\hline $\begin{array}{l}\text { \% de participantes que camina o va en bici- } \\
\text { cleta en sus desplazamientos semanales }\end{array}$ & 88,6 & 76,3 & 77,7 & 76,2 & 62,7 & 77,3 \\
\hline $\begin{array}{l}\text { \% Días por semana en que se desplaza ca- } \\
\text { minando o en bicicleta (0-1 / 2-3 / } 4 \text { o más) }\end{array}$ & $\begin{array}{c}12,6 / \\
20,2 / 67,2\end{array}$ & $\begin{array}{c}22,8 / \\
21,2 / 56\end{array}$ & $\begin{array}{c}25,9 / \\
23,4 / 50,7\end{array}$ & $\begin{array}{c}22,2 / \\
22,2 / 55,6\end{array}$ & $\begin{array}{c}35,2 / \\
11,7 / 52,9\end{array}$ & $\begin{array}{c}22,9 / \\
20,4 / 56,7\end{array}$ \\
\hline $\begin{array}{l}\text { Encuestados que dicen que estado de salud } \\
\text { es mejor o mucho mejor que hace un año (\%) }\end{array}$ & 69,6 & 72,4 & 65,4 & 58,7 & 78,4 & 69,1 \\
\hline
\end{tabular}

Fuente: elaboración de los autores.

Andrés Bello, donde las personas declararon estar 136 y 127 min respectivamente.

El 77,3\% de los encuestados declararon moverse rutinariamente en forma activa (bicicleta o caminata) en sus desplazamientos cotidianos, mientras que 56,7\% de los participantes dijo hacerlo activamente al menos cuatro días a la semana. Los valores máximos se encontraron en la CicloRecreoVía de Santiago Centro (67,2\%), y los mínimos en la de Pocuro (50,7\%). Finalmente, $69,1 \%$ de los encuestados declararon sentirse tener un estado de salud mejor o mucho mejor que el de hace un año.

Dentro de las motivaciones para usar la CicloRecreoVía, 32\% de los participantes aseguraron hacerlo para realizar actividad física, $31 \%$ para recrearse, $15 \%$ por razones de salud, y $12 \%$ para conocer más la ciudad y estar al aire libre. El resto de los encuestados declaró otras motivaciones.

\section{Discusión}

Los resultados de esta investigación mantienen varios puntos de concordancia con investigaciones internacionales. En primer lugar, el tiempo promedio que las personas pasan en la CicloRecreoVía (113 min), guarda relación con lo registrado en investigaciones internacionales en esta materia ${ }^{16,18}$, y sugiere que ejercitarse semanalmente en las CicloRecreoVías ayudaría a cubrir buena parte los 150 min semanales de AF de tipo moderado recomendados por la Organización Mundial de la Salud ${ }^{7}$, aunque lo recomendable es que la actividad física no sea realizada en una vez a la semana sino distribuida a lo largo de la semana. De igual manera, los resultados encontrados en esta investigación son consistentes con los reportados internacionalmente $e^{18,19,21}$, que muestran que los usuarios de este tipo de programas tienden a llevar modos de vida activos en sus rutinas diarias.

En segundo lugar, los datos relativos a las motivaciones de los usuarios para participar en el programa son también concordantes con investigaciones internacionales ${ }^{21,26}$, mostrando que la causas principales para ser parte de este evento son realizar actividad física de tipo recreativo, el cuidado de la salud y la entretención, expresada en conocer la ciudad de una manera diferente. Los altos índices de auto-percepción del estado de salud de los participantes de las CicloRecreoVías recolectados en este estudio podrían estar señalando que el programa esta ayudando a que ellos percibieran de mejor manera su calidad de vida, tal como lo señalan investigaciones llevadas a cabo en Colombia ${ }^{21}$.

En tercer lugar, los resultados sugieren que la disponibilidad de infrastructura y programas para la realización de AF en la ciudad esta positivamente asociada a la adopción de modos de vida activos en la población, cuestión reportada en investigaciones chilenas recientes ${ }^{8}$. Futuras investigaciones debieran abordar la causalidad de este comportamiento, esto es, si el uso de la CicloRecreoVía (o los gimnasios al aire libre en el caso de la investigación antes citada), es una 
consecuencia o una causa de la adopción de rutinas activas en las personas, como irse al trabajo caminando o en bicicleta.

En cuarto lugar, se detectó que las CicloRecreoVías parecen atraer a distintos tipos de participantes en función de su ubicación en la ciudad. Mientras las CicloRecreoVías de sectores centrales convocaron a personas de comunas muy diversas socialmente y alejadas del centro, las ubicadas en zonas residenciales no centrales (Irarrázaval y La Florida), convocaron principalmente a residentes de la misma comuna. En este sentido, los resultados sugieren que más allá de los aportes reportados en la actividad física ${ }^{20}$, la promoción del uso de la bicicleta y la caminata ${ }^{19}$, o la creación de capital social en los entornos al permitir la ocupación del espacio público por las personas ${ }^{19}$, la CicloRecreoVía estaría también contribuyendo a la cohesión social de las ciudades. Esto porque permitiría que personas de comunas social y geográficamente distantes hagan uso del espacio metropolitano (en el caso de las CicloRecreoVías centrales), al tiempo de facilitar el encuentro vecinal (en el caso de las CicloRecreoVías no centrales). Futuras investigaciones debieran ahondar en estas ideas.

En síntesis, en concordancia con los resultados de investigaciones previas ${ }^{16,19,21,27}$ los resultados indican que la CicloRecreoVía tiene el potencial de generar resultados positivos de salud publica en los habitantes, por lo que debieran ser incorporadas a las políticas de salud preventivas del Ministerio de Salud. Futuras líneas de investigación en este tema debieran analizar con mayor profundidad aspectos como la intensidad de la actividad física realizada por los participantes durante la CiclorecreoVía, la adherencia que tienen estos programas en el tiempo y bajo distintas condiciones climáticas, y los efectos de este tipo de programas en el cambio de hábitos alimenticios o la disminución de la obesidad.

\section{Referencias}

1. Ministerio de Salud, Gobierno de Chile. Encuesta Nacional de Salud ENS Chile 2009-10. Accesado desde http://web.minsal.cl/portal/url/item/bcb03d7bc28b64dfe040010165012d23.pdf.

2. Organización de las Naciones Unidas para la alimentación y la agricultura / Organización Panamericana de la Salud (2016). FAO. Panorama de la Seguridad Alimen- taria y Nutricional de América Latina 2016, Accesado desde: http://www.fao.org/3/a-i6747s.pdf.

3. Ministerio de Salud, Gobierno de Chile. Orientaciones para planes comunales de promoción de la salud 2014. Accesado desde http://www.minsal.cl/sites/default/files/ orienplancom2014.pdf.

4. Gobierno de Chile, Ministerio de Salud, Estrategia Global Contra la Obesidad (2006). Accesado desde: http:// www.pamchile.cl/evento/evento03/pre02d.pdf.

5. www.eligevivirsano.cl

6. Gobierno de Chile, Elige vivir sano. Metas Sanitarias 2011-2020. Accesado desde http://web.minsal.cl/portal/ url/item/c4034eddbc96ca6de0400101640159b8.pdf.

7. Organización Mundial de la Salud, OMS. Recomendaciones mundiales sobre la actividad física para la salud. 2010. Accesado desde: http://www.who.int/dietphysicalactivity/strategy/eb11344/strategy_spanish_web.pdf.

8. Organización Mundial de la Salud. OMS. Estrategia Mundial sobre dieta, actividad física y salud, 2004. Accesado desde: http://apps.who.int/iris/bitstre am/10665/44441/1/9789243599977_spa.pdf.

9. Ibarra M, Mora R. Habitar la escuela: El problema de la infraestructura y su relación con las enfermedades escolares en Chile. Rev INVI 2011; 26 (71): 109-31.

10. Mora R, Weisstaub G, Greene M, Herrmann G. Outdoor gyms in Santiago: urban distribution and effects on hysical activity. Motriz 2017; 22 (4):1-7. doi: http:// dx.doi.org/10.1590/S1980-6574201600040002.

11. Sallis JF, Cerin E, Conway TL, Adams M, Frank LD, Pratt $\mathrm{M}$, et al. Physical activity in relation to urban environments in 14 cities worldwide: a cross-sectional study. The Lancet 2016, 387. Accesado desde: http:// www.thelancet.com/pdfs/journals/lancet/PIIS01406736(15)01284-2.pdf.

12. Cervero R, Kockelman K. Travel demand and the 3DS: density, diversity, and design. Transport Res D: TR E 1997; 2 (3): 199-219.

13. USA Task Force on Community Preventive Services. Increasing Physical Activity. A Report on Recommendations of the Task Force on Community Preventive Services, 2001. Accesado desde: https://www.cdc.gov/ mmwr/preview/mmwrhtml/rr5018a1.htm.

14. Jacoby E, Pardo CF. Ciudades del automovil, obesidad y cambio climatico. Slow Research. Accesado desde http:// www.bvsde.paho.org/texcom/cd045364/ciudadesauto. pdf.

15. Montezuma R. The transformation of Bogotá, Colombia, 1995-2000: investing in citizenship and urban mobility. Glob Urb Dev 2005; 1: 1-10.

16. Sarmiento OL, Díaz del Castillo A, Triana CA, Acevedo MJ, González SA, Pratt M. Reclaiming the streets 
for people: Insights from Ciclovías Recreativas in Latin America. Prev Med 2016. doi:10.1016/j.ypmed.2016.07.028.

17. Montero S. Worlding Bogotá's Ciclovía From Urban Experiment to International "Best Practice." Lat Am Perspect 2017, 40 (2): 135-45. doi: https://doi.org/10.1177/0094582X16668310.

18. Torres A, Sarmiento OL, Stauber C, Zarama R. The Ciclovia and Cicloruta programs: promising interventions to promote physical activity and social capital in Bogotá, Colombia. Am J Public Health 2013; 103 (2): e23-e30. doi: 10.2105/AJPH.2012.301142.

19. Hipp JA, Eyler AA, Kuhlberg JA. Target Population Involvement in Urban Ciclovias: A Preliminary Evaluation of St. Louis Open Streets. J Urban Health 2012. Accesado desde: http://openscholarship.wustl.edu/cgi/ viewcontent.cgi? article $=1013 \&$ context $=$ brown_facpubs.

20. Engelberg JK, Carlson JA, Black ML, Ryan S, Sallis, JF. Ciclovía participation and impacts in San Diego, CA: The first CicloSDias. Prev Med 2014; 69 Supp: S66-S73.

21. Sarmiento OL, Schmid TL, Parra DC, et al. Quality of life, physical activity, and built environment characteristics among colombian adults. J Phys Act Health 2010; 7
(Suppl 2): S181-95.

22. Montes F, Sarmiento OL, Zarama R, Pratt M, Wang G, Jacoby E, et al. Do Health Benefits Outweigh the Costs of Mass Recreational Programs? An Economic Analysis of Four Ciclovía Programs. J Urban Health 2012; 89( 1): 153-70. doi: 10.1007/s11524-011-9628-8.

23. www.ciclorecreovía.cl

24. http://activelivingresearch.org/open-streets-initiatives-measuring-success-toolkit

25. Organización Mundial de la Salud. Cuestionario Mundial sobre Actividad Física (GPAQ) Accesado desde: http://hhs.sdsu.edu/wp-content/uploads/2012/06/ gpaq-spanish.pdf.

26. Zieff SG, Kim MS, Wilson J, Tierney P. A “Ciclovia” in San Francisco: Characteristics and physical activity behavior of Sunday Streets participants. J Phys Act Health. 2014; 11 (2): 249-55. doi: 10.1123/jpah.2011-0290. Epub 2013 Jan 30.

27. Díaz del Castillo A, Pedraza, CM, González SA, Díaz J, Ibarra L, Fernández D, et al. Ciclovías Recreativas: Una epidemia saludable 2013. Accesado desde; http:// epiandes.uniandes.edu.co/wp-content/uploads/FINAL_FactSheet_CicloviasRecreativas_ENG_15.05.13. pdf. Published 2013. 